\title{
On the orbital period changes of two W-type overcontact binaries: RW Comae Berenices and CE Leonis
}

\begin{abstract}
Qian Shengbang*
Yunnan Observatory, Chinese Academy of Sciences, PO Box 110, 650011 Kunming, PR China

National Astronomical Observatories, Chinese Academy of Science (NAOCAS)

United Laboratory of Optical Astronomy, Chinese Academy of Science (ULOAC)

Received 14 May 2001 / Accepted 30 November 2001

Abstract. $\mathrm{O}-\mathrm{C}$ diagrams of two very short-period W-type overcontact binaries, RW Com and CE Leo, are formed by considering the general trends of their $\mathrm{O}-\mathrm{C}$ curves and the natures of the period changes are analyzed. For RW Com, its period shows a secular decrease with a rate of $\mathrm{d} P / \mathrm{d} t=-6.06 \times 10^{-8}$ days/year. Weak evidence indicates that a small-amplitude oscillation is superimposed on the secular decrease. For CE Leo its period is increasing at rate of $\mathrm{d} P / \mathrm{d} t=+7.20 \times 10^{-7}$ days/year. The masses of the primary components of the two W UMa stars are nearly the same and the main difference between the two systems is that they have a different mass ratio ( $q=0.34$ for RW Com and 0.50 for CE Leo respectively). The different type of period changes may be the result of their different mass ratio. These findings are in agreement with Qian's (2001) conclusion that W-type stars with high-mass ratio $(q>0.4)$ usually show a increasing period, while the periods of low-mass ratio systems $(q<0.4)$ are decreasing. The period oscillation of RW Com may be caused either by the presence of an unseen third body or by magnetic activity cycles. The secular period changes of the two binary stars can be explained by the combination of the thermal relaxation oscillation (TRO) and the variable angular momentum loss (AML) via the change of depth of overcontact.
\end{abstract}

Key words. stars: binaries: close - stars: individual: RW Com - stars: individual: CE Leo - stars: evolution

\section{Introduction}

RW Com was discovered to be a W UMa type star by Jordan (1923). It was later studied by others (for references see Milone et al. 1980). A series of works by Milone et al. (1980, 1985, 1987) showed that RW Com is an W-type overcontact binary with a mass ratio of $q=0.34$. The light variation of CE Leo was discovered by Hoffmeister (1963) who classified the variable as RRctype. Wenzel \& Zeigler (1966) identified the system as a W UMa-type binary and published the first ephemeris: Min.I $=2437651.650+0.3034286 \times E$. Later, CE Leo was observed photoelectrically by Hoffmann (1983) and by Samec \& Bookmyer (1987). Recently, Samec et al. (1993) published complete light curves in BVRI and a photometric analysis of their observations based on the WD (Wilson-Devinney) method. It was shown that CE Leo is a W-type overcontact binary with a mass ratio of 0.51 . The physical parameters of the two W UMa stars, given by Maceroni and van't Veer (1996), are listed in Table 1 in solar units.

\footnotetext{
* e-mail: qsb@netease.com
}

\section{Variations in the period of RW Com}

Epochs and orbital periods of RW Com have been given by several authors (for references see Srivastava 1987). The period change of the system was first noticed by Milone et al. (1980) who gave a period decrease rate of $0.4 \times 10^{-10}$ day per day, but did not present an $\mathrm{O}-\mathrm{C}$ diagram. Many times of light minimum of RW Com were collected by Srivastava (1987) and after his collection some visual or photographic timings were compiled in the Eclipsing Binaries Minima Database (EBMD) (available at http://www.oa.uj.edu.pl/ktt/krttk_dn.html) and two photoelectric timings have been published by Ogloza (1997). The O-C diagram calculated with the ephemeris (Beljawsky 1924): Min.I $=2419127.234+0 \mathrm{~d} 237350 \times E$ is presented in Fig. 1. It has been split into several portions by Srivastava (1987) who proposed that several period jumps have occurred around points B, C and D. However, these jumps were mainly based on two visual timings and from points $\mathrm{C}$ to $\mathrm{D}$ the $\mathrm{O}-\mathrm{C}$ values suddenly increase 0.127 in a short time interval (about 6700 days). Before and after the jump, the general $\mathrm{O}-\mathrm{C}$ trend shows secular decrease. These facts indicate that the $\mathrm{O}-\mathrm{C}$ jumps 
Table 1. Physical properties of two overcontact binaries.

\begin{tabular}{lllllllll}
\hline star name & $M_{1}$ & $M_{2}$ & $R_{1}$ & $R_{2}$ & $P$ & $i$ & $f$ & $q$ \\
\hline RW Com & 0.92 & 0.31 & 0.84 & 0.52 & 0.237 & $75 .{ }^{\circ} 2$ & 17 & 0.34 \\
CE Leo & 0.94 & 0.47 & 0.94 & 0.68 & 0.303 & $84 . .^{\circ} 6$ & 3 & 0.50 \\
\hline
\end{tabular}

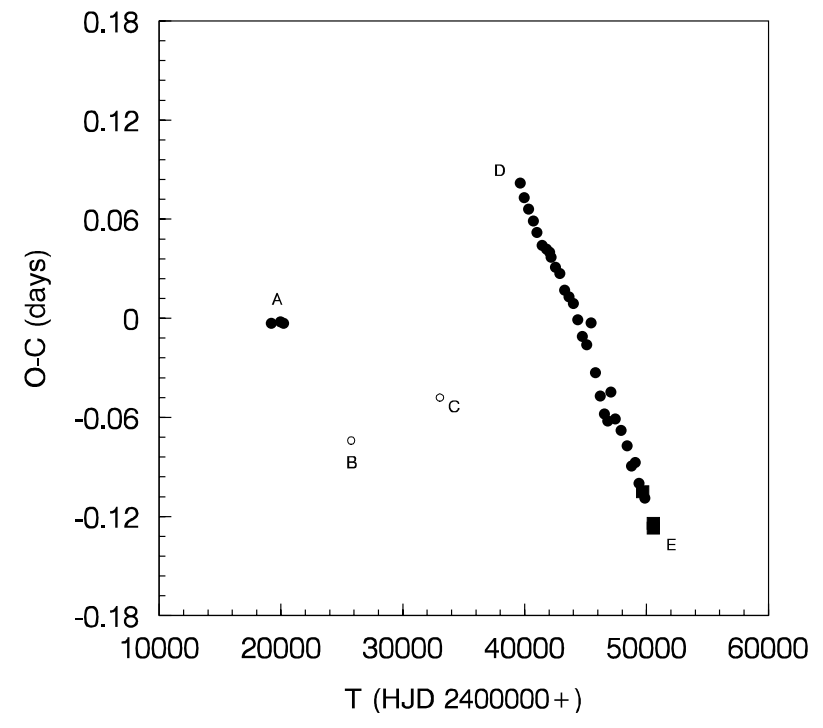

Fig. 1. O-C diagram of RW Com. Solid dots refer to mean $\mathrm{O}-\mathrm{C}$ values of some visual or photographic data, solid squares to photoelectric observations, and two open circles to individual visual timings.

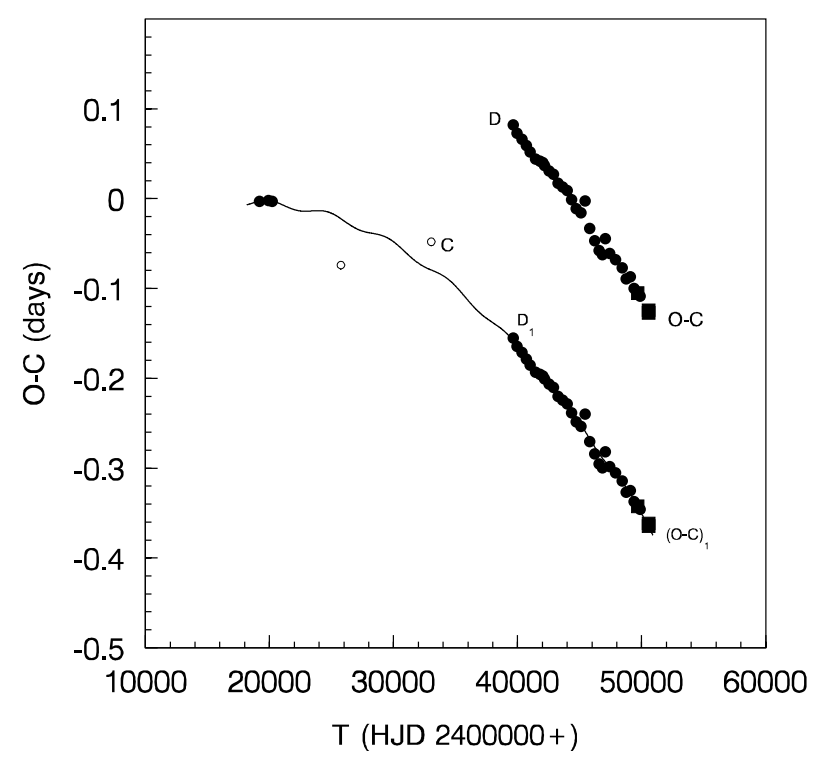

Fig. 2. O-C curve of RW Com formed by considering the general $\mathrm{O}-\mathrm{C}$ trend.

may be caused by the miscalculation of the $\mathrm{O}-\mathrm{C}$ values of timings after HJD 2438000.

The new $(\mathrm{O}-\mathrm{C})_{1}$ curve formed is displayed in Fig. 2 which may show a roughly parabolic variation indicating a secular decrease in the period. Srivastava (1987) pointed out that the data in the time interval 1967-1986 showed a sinusoidal variation. In order to check this, a sinusoidal

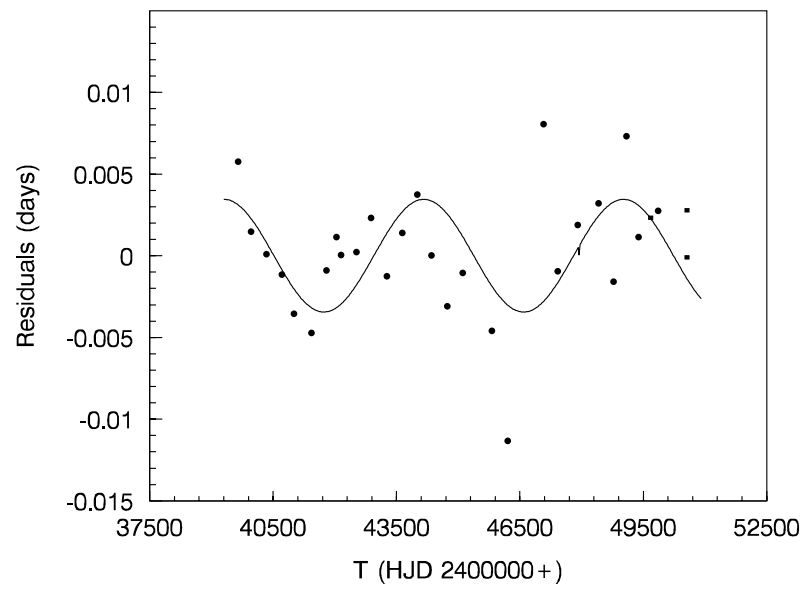

Fig. 3. Residuals of timings after HJD 2438000 calculated with the quadratic part of Eq. (1)

term is added to a quadratic ephemeris to get a good fit to the observations (solid line in Fig. 2):

$$
\begin{aligned}
\text { Min.I }= & 2419127.2290(33)+0.23734990(10) \times E \\
& -1.97(7) \times 10^{-11} \times E^{2} \\
& +0.0035(13) \sin \left(0.0176 \times E+35^{\circ}(12)\right)
\end{aligned}
$$

During the least-squares solution, the two individual visual timings are not used. With the coefficient of the square term, a continuous period decrease rate $\mathrm{d} P / \mathrm{d} t=$ $-6.06 \times 10^{-8}$ days/year is determined.

The residuals of the timings after HJD 2438000 based on the quadratic part of Eq. (1), are presented in Fig. 3 where two points showing large deviations are discarded. Although they show slightly large scatters, the general trend of those residuals may indicate a cyclic variation. With the periodic part of Eq. (1), a cyclic variation with a period of about $T=13.3$ years and an amplitude of about $A=0.0035$ is determined which can be explained by the light time effect via the presence of a third body. If the orbital plane of the third body is parallel to the visual line, its mass should be: $M_{3}=0.133 M_{\odot}$. On the other hand, RW Com contains two late-type stars which possess strong magnetic activity. The period oscillation may be caused by magnetic activity cycles in the components.

\section{Variation in the period of CE Leo}

Many times of light minimum of CE Leo were tabulated in the paper of Samec et al. (1993). After their collection, some timings have been reported in the EBMD and three CCD times have been published by Safar \& Zejda (2000). The O-C values calculated with the ephemeris of 


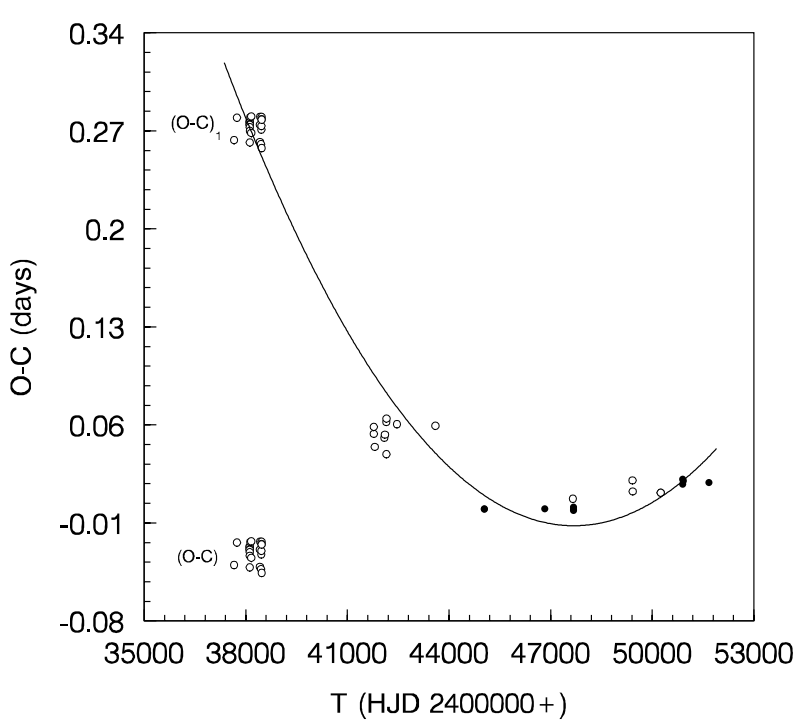

Fig. 4. O-C curve of CE Leo where open circles refer to visual or photographic observations and solid dots to photoelectric or CCD data.

Samec et al. (1993): Min.I $=2447679.6689+0$ d $30342785 \times$ $E$ are shown in Fig. 4. In order to understand the nature of the period change of CE Leo easily, 10 visual timings published in earlier BBSAG bulletins Nos. 9-37 have been omitted by Samec et al. (1993) and they proposed that the period showed an abrupt or a continuous decrease. However, the $\mathrm{O}-\mathrm{C}$ values $(0.0213,0.0177$, and 0.0201$)$ of the three CCD timings show that recently the period of CE Leo has been increasing.

As we can see from Fig. 4, the general $\mathrm{O}-\mathrm{C}$ trend after HJD 2441000 suggests that the orbital period of $\mathrm{CE}$ Leo may show secular increase. By considering this general trend, $\mathrm{O}-\mathrm{C}$ values of Meinunger \& Wenzell's (1968) timings are recalculated and are shown in Fig. 4 as $(\mathrm{O}-\mathrm{C})_{1}$. With weight 1 for visual or photographic data and weight 8 for photoelectric or CCD observations, a weighted least-squares solution leads to the following ephemeris:

$$
\begin{aligned}
\text { Min.I }= & 2447679.6575(27)+0.30342826(1) \times E \\
& +2.99(2) \times 10^{-10} \times E^{2} .
\end{aligned}
$$

The coefficient of the square term reveals a continuous period increase rate of $\mathrm{d} P / \mathrm{d} t=+7.20 \times 10^{-7}$ days/year. If only the photoelectric and CCD data are used, we can obtain:

$$
\begin{aligned}
\text { Min.I }= & 2447679.66939(9)+0.30342868(4) \times E \\
& +9.30(63) \times 10^{-11} \times E^{2}
\end{aligned}
$$

and a period increase rate of $\mathrm{d} P / \mathrm{d} t=+2.24 \times$ $10^{-7}$ days/year. Although the rate is smaller than that derived using all timings, the general trends of both are the same.

\section{Discussions and conclusions}

If the secular period changes of the two W UMa stars are caused by a conservative mass transfer (MT) between the components, then the mass transfer rates would be: $\mathrm{d} M / \mathrm{d} t=3.98 \times 10^{-8}$ and $7.45 \times 10^{-7} M_{\odot} /$ year but with different directions. The masses of the primary components of RW Com and CE Leo are almost equal. Why do their periods show different changes? One is decreasing, while the other is increasing. As shown in Table 1, the main difference in the two systems is the slightly larger mass ratio. This suggests that the different mass ratios may result in the different kind of period changes. Recently, based on period changes of $30 \mathrm{~W}$-type overcontact binaries, Qian (2001) has found that there may exist a correlation between the period change and the mass ratio. The high-mass ratio systems $(q>0.4)$ usually show secular period increase, while the orbital periods of lowmass ratio systems $(q<0.4)$ vary with a long-time decrease. The period changes of the two $\mathrm{W}$-type systems are in agreement with this conclusion.

Secular period variations for W UMa stars, especially decreases, are usually explained by angular momentum loss (AML) via magnetic stellar wind. However, for RW Com and CE Leo, the timescales of the period changes are $3.9 \times 10^{6}$ and $4.2 \times 10^{5}$ years respectively that are much shorter than the timescale of the AML (about $10^{8}-10^{10}$ years). How can the AML cause the secular period change? In order to explain this, Qian (2001) proposed an artificial scenario in which the rate of AML is changed by the variation of the depth of overcontact. For a given W UMa star, the period decrease means the reduction of separation between the components and the increasing depth of overcontact. This will cause increased mixing in the CCE which tends to bury the strong surface magnetic field. Thus, the magnetic braking is weakened, leading to a decreased AML rate (Vilhu 1981; Smith 1984). As the rate of AML is less than the critical loss rate of Rahunen (1981), the calculations of Rahunen (1981) have shown that the evolution of the system is mainly controlled by thermal relaxation oscillation (TRO) (Lucy 1976; Flannery 1976; Robertson \& Eggleton 1977). The period will increase and then decrease the depth of overcontact. Then, the decreased mixing in the CCE may result in a rather strong magnetic field which causes a large AML (larger than the critical value of Rahunen 1981). In this case, the evolution of the system is mainly controlled by AML, and finally, the period will decrease again. The different period changes of the two systems may suggest they are at different evolutionary stages, i.e., RW Com is on the AML-controlled stage, while CE Leo is on the TRO-Controlled stage.

Acknowledgements. This work was supported by the Chinese Natural Science Foundation (No. 10003004) and by the National Key Fundamental Research Project through grant G1999075405. Many thanks are given to the referee for his/her useful suggestions and comments and to the copy-editor for improving the author's English writing. 


\section{References}

Beljawsky, S. 1924, Astr. Nach., 221, 139

Flannery, B. P. 1976, APJ, 205, 217

Hoffmann, M. 1983, IBVS, 2344

Hoffmeister, C. 1963, AN, 287, 169

Jordan, F. C. 1923, AJ, 35, 44

Lucy, L. B. 1976, ApJ, 205, 217

Maceroni, C., \& van't Veer, F. 1996, A\&A, 311, 523

Meinunger, L., \& Wenzel, W. 1968, VSS(4), 430

Milone, E. F., Chia, T. T., Castle, K. G., Robb, R. M., \& Merrill, J. E. 1980, ApJS, 43, 339

Milone, E. F., Hrivnak, B. J., Hill, G., \& Fisher, W. A. 1985, AJ, 90, 109
Milone, E. F., Wilson, R. E., \& Hrivnak, B. J. 1985, ApJ, 319, 325

Ogloza, W. 1997, IBVS, 4534

Qian, S. B. 2001, MNRAS, 328, 635

Rahunen, T. 1981, A\&A, 102, 81

Robertson, J. A., \& Eggleton, P. P. 1977, MNRAS, 179, 359

Safar, J., \& Zejda, M. 2000, IBVS, 4888

Samec, R. G., \& Bookmyer, B. B. 1987, IBVS, 3053

Samec, R. G., Wen Su, Terrell, D., \& Hube, D. P. 1993, AJ, 106,318

Smith, R. C. 1984, Q. Jl R. Astro. Soc., 25, 405

Srivastava, R. K. 1987, Ap\&SS, 139, 373

Vilhu, O. 1981, Ap\&SS, 78, 401

Wenzel, W., \& Zeigler, E. 1966, MVS, 4(2), 20 